\title{
Common pitfall of plastic surgeon for diagnosing cutaneous odontogenic sinus
}

\author{
Lan Sook Chang \\ Department of Plastic and \\ Reconstructive Surgery, Inje University \\ Ilsan Paik Hospital, Inje University \\ College of Medicine, Goyang, Korea
}

\begin{abstract}
Dental origins are a common cause of facial cutaneous sinus tracts. However, it can be easily overlooked or misdiagnosed if not suspected by a surgeon who is not familiar with dental origins. Cutaneous odontogenic sinuses are typically nodulocystic lesions with discharge and are most frequently located on the chin or jaw. This article presents two cases of unusual cutaneous odontogenic sinus presentations, as deep dimpling at the middle of the cheek. The patients were undergone surgical excision of sinus tract and dimpling immediate before and after treatment of causal teeth and the lesions resolved without recurrence. Surgeons should consider dental origins of facial dimpling lesions with discharge and provide appropriate treatment.
\end{abstract}

Keywords: Facial dimpling / Cutaneous fistula / Periapical abscess

\section{INTRODUCTION}

Patients with skin lesions on the face who require surgery are usually referred to plastic surgeons. Skin lesions may vary in mass, ulceration, discoloration, the presence of dimples, and other features. Among these factors, dimples are uncommon and are induced by skin retraction. Dimples can be associated with protuberant dermatofibrosis, sinus tracts of cyst, ulcerated dimples of carcinoma [1]. Surgeons would face embarrassment if the skin lesions recur or persist after attempting to excise a dimple, under the suspicion of the aforementioned lesions.

Cutaneous odontogenic sinus, one of the important differential diagnosis of facial sinus lesions, is a disease that is easily overlooked by doctors, but not by dentists, despite reports throughout the literature [2-9]. Excision of the skin lesion alone and neglecting the dental etiology can result in recurrence or persistence of wound. [2]. A cutaneous odontogenic sinus is

\footnotetext{
Correspondence: Lan Sook Chang

Department of Plastic and Reconstructive Surgery, Inje University Ilsan Paik Hospital, Inje University College of Medicine, 170 Juhwa-ro, Ilsanseo-gu, Goyang 10380, Korea

E-mail: changls02@gmail.com
}

Received September 4, 2018 / Revised October 3, 2018 / Accepted October 16, 2018 typically a nodulocystic lesion, and $80 \%$ of such lesions are most frequently located on the chin or jaw [3]. If the location and characteristics are atypical, this condition is more likely to be misdiagnosed. We report two cases of unusual cutaneous odontogenic sinus presentations that involved deep dimpling at the middle of the cheek. The purpose of this report is to increase the awareness of inexperienced plastic surgeons of the dental origins of suspicious facial dimpling lesions.

\section{CASE REPORTS}

\section{Case 1}

A 23-year-old man presented with a dimple on his left cheek (Fig. 1). He had been treated for 1 year at a local clinic with three episodes of surgical excision, under the suspicion of an epidermal cyst, which recurred immediately after excision. He complained of recurrent pus drainage from the lesion, but the opening was closed and pus could not be found upon squeezing when he visited our center. On physical examination, a cord-like tissue was palpable, and he had no tenderness anywhere on palpation. Based on his history and clinical appearance, a diagnosis of sinus tract of epidermal cyst was consid- 

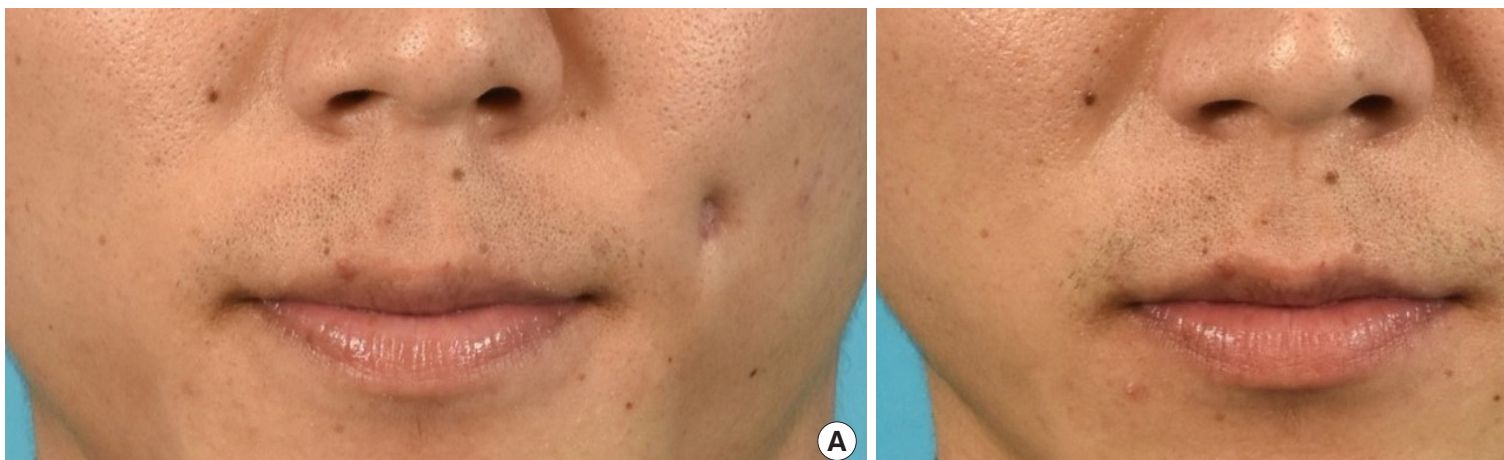

A

Fig. 1. A 23-year-old man with a dimple on his left cheek. (A) Preoperative photo. (B) Three-month postoperative photo.
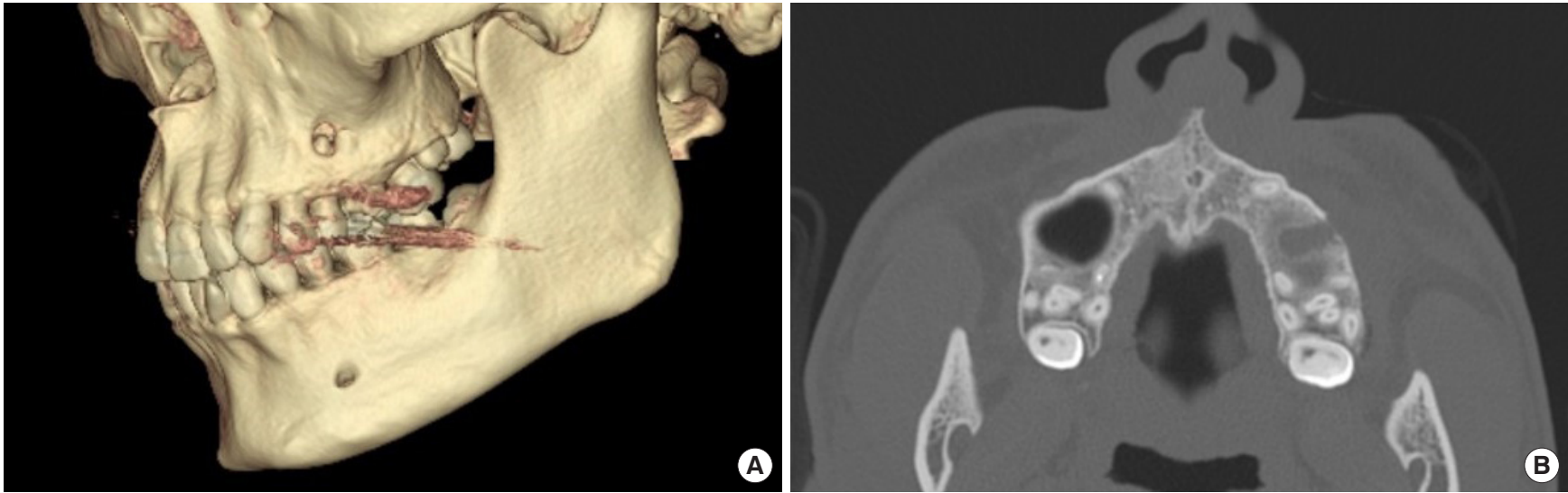

Fig. 2. Computed tomography images showing a focal bony cortical defect at the left upper second premolar root, with a periapical abscess. (A) Three-dimensional reconstruction view. (B) Axial view.
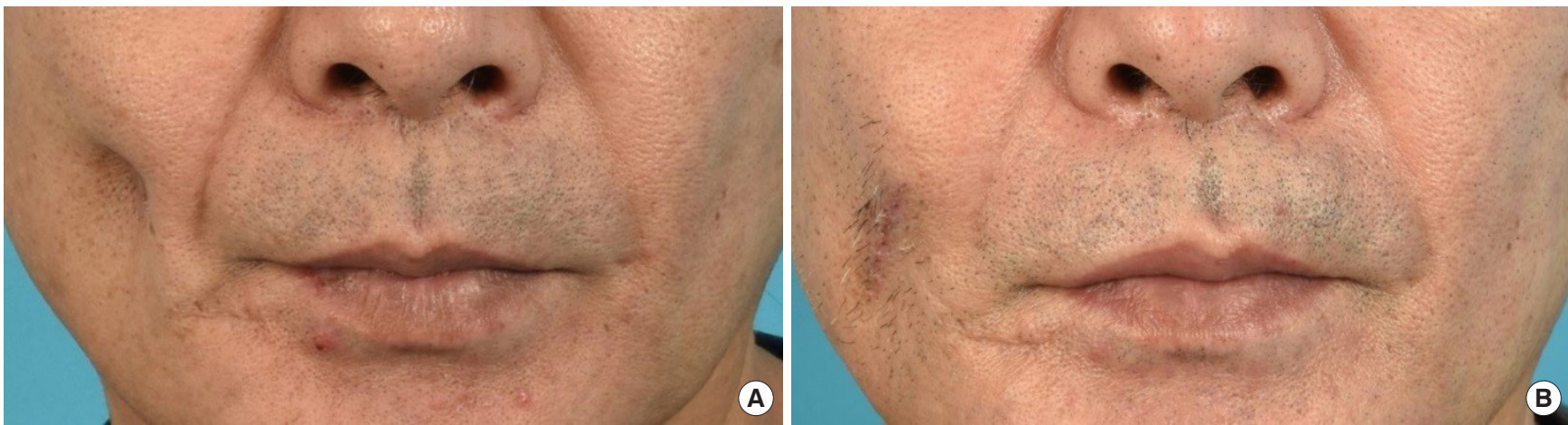

Fig. 3. A 55-year-old man with a deep dimple on his right cheek. (A) Preoperative photo. (B) Three-month postoperative photo.

ered. The wide excision revealed a cord-like tissue that connected the skin to the maxilla periosteum. All inflamed and contracted tissue was excised, and the initial histological examination revealed chronic inflammation with extensive fibrosis, without evidence of a cyst. After 6 days after operation, wound dehiscence was found with discharge. A computed tomography (CT) evaluation showed a focal bony cortical defect at the left upper second premolar root, with a periapical abscess (Fig. 2). He was suspended while undergoing endodontic treatment of the left upper second premolar, and he had neither dental attention nor dental pain. The tooth was extracted by a dentist. Necrotic tissue around the sinus was removed, and the contracted skin was undermined for the wound closure 5 days after the extraction. No recurrence was found postoperatively until after 1 year.

\section{Case 2}

A 55-year-old man with no treatment history presented a deep 


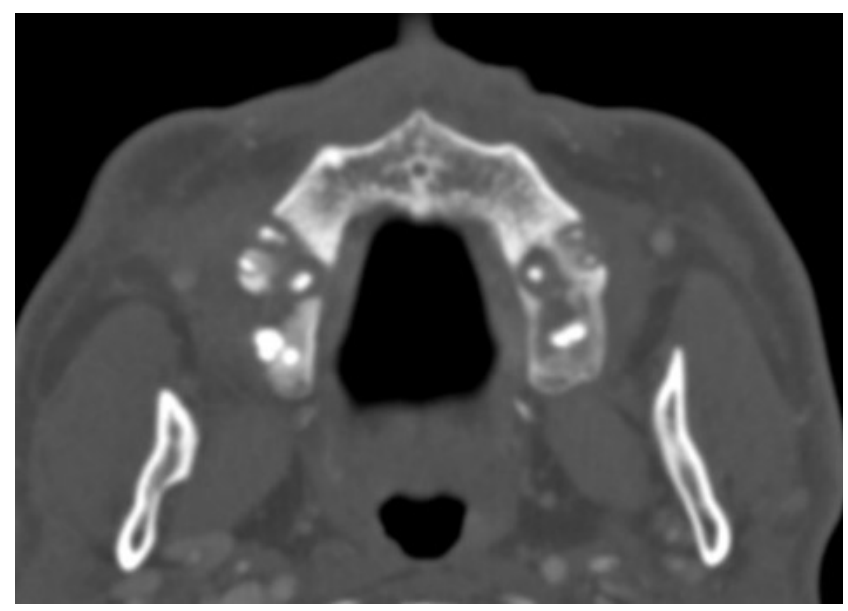

Fig. 4. Computed tomography image showing multiple periodontitis at bilateral upper molar teeth.

dimple on his right cheek (Fig. 3). The lesion began as a pustule 1 month ago. After spontaneous rupture, the skin was retracted and pus continuously drained through the opening. On physical examination, a small nodule was fixed to the underlying bone, and cord-like hard tissue was palpable under the nodule, which caused the deep skin retraction. Pus drained though the opening upon squeezing. He denied any intraoral problem, and the initial differential diagnosis was between an epidermal cyst and squamous cell carcinoma. Underlying structures were evaluated using CT, which revealed multiple periodontitis at bilateral upper molar teeth, with odontogenic sinusitis in both maxillary sinuses and no definite cystic component (Fig. 4). He did not have any dental pain or symptoms. Despite that we recommended periodontitis treatment first, he requested surgery for his skin lesion first. The nodule was excised, and cord-like tissue that connected the nodule to the maxilla periosteum was observed. After resection of the sinus, an alveolar bony defect was found (Fig. 5). A massive irrigation was performed and the contracted skin was undermined to close the wound. The histologic examination revealed chronic inflammation with fibrosis. The following day, he began endodontic treatment, and the cutaneous wound healed completely without recurrence for 1 year postoperation.

\section{DISCUSSION}

Cutaneous sinus tracts in the lower face can be caused by infectious disease such as mycobacterial infections, actinomycosis, deep fungal infections, osteomyelitis, or tumors such as pyogenic granulomas, epidermal cysts, squamous cell carcinomas, or congenital malformations such as thyroglossal duct cysts, brachial cleft cysts, and salivary gland fistulas [4]. A dental ori-

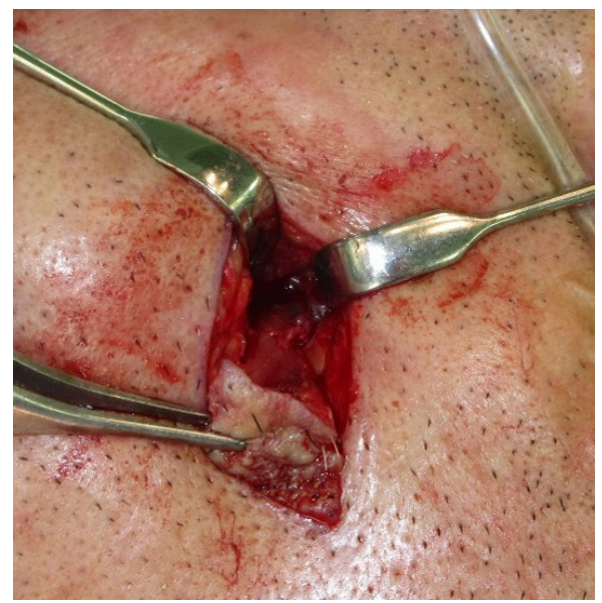

Fig. 5. Intraoperative photo showing the alveolar bony defect after resection of the sinus.

gin is important in the differential diagnosis of cutaneous sinus tracts because correct diagnoses allow prompt resolution of the lesion, while misdiagnoses can delay treatment and lead to facial deformity [5].

Chronic periodontitis is the main cause of cutaneous odontogenic sinus tracts and is usually secondary to dental caries. Pulpal degeneration secondary to trauma is also a common etiologic factor [6]. Periapical inflammation can spread along the path of least resistance and establish an intraoral or extraoral sinus tract. While the prevalence of intraoral sinus tracts has been reported as almost one in five teeth with periradicular inflammation, extraoral sinus is relatively rare and reported as a case series [7]. In addition, patients with cutaneous odontogenic sinuses who are unaware of their condition are more likely to seek plastic surgeon or dermatologist, non-dentist, although this lesion is well described in the dental literature. This can lead cutaneous odontogenic sinuses to be regularly overlooked and mismanaged [8].

The cutaneous portion of odontogenic sinuses are typically nontender, erythematous, symmetrical, and small nodulocystic lesions. On physical examination, cords of tissue that connect the skin to the maxilla or mandible can be palpable. Squeezing of the cord can excrete pus-like discharge though the sinus opening as a small central punctum [2]. Perilesional skin can be slightly retracted producing dimpling. The origin of the maxillary teeth, as in the patients in this report, is more noticeable, which deepens the dimpling due to the abundance of soft tissue.

Eighty percent of reported cases are associated with mandibular teeth and remainings are maxillary teeth [2]. Consequently, the most common sites for cutaneous odontogenic sinuses are the chin and the jaw [3]. Other areas have also been reported to 
have cutaneous odontogenic sinuses, such as the cheek [9], inner canthus of the eye [10], intranasal areas [11], and nasolabial folds [2]. Most teeth roots are closer to the lateral cortex of the jaw, and infections tend to spread from the apex to the buccal plate, rather than to the palatal plate [6]. Once the cortical plate has been penetrated, the sinus tract exit is identified based on the relationship between the muscle attachment location and the tooth apex [9]. If the maxillary teeth root apex is beyond the buccinator muscle attachment site, the exit is made to penetrate the skin, while the exit is located intraorally, with maxillary teeth below the muscle. [6].

Panoramic X-rays or periapical dental films are very informative for diagnosing cutaneous odontogenic sinuses, through the radiolucency of the offending teeth [12]. These images can usually be obtained through collaborations with dentists, and the procedure is not easy to perform, unless a dental origin is initially suspected. CT can greatly contribute to the evaluation of the cause and extent of infection when the diagnosis is unclear. Odontogenic infections can be seen in CT images as a bony changes, such as an interruption or thinning of the alveolar cortical plate that surrounds the causal tooth, soft tissue changes such as thickening of the muscles and disappearance of the fat layer between the muscles, and paranasal sinus changes such as mucosal thickening, fluid accumulation, and bony wall thickening [13].

Awareness that the cause of a facial dimpling lesion with discharge could be of dental origin is crucial for a correct diagnosis. Patients may not recall whether they had a toothache due to the low grade and insidious nature of odontogenic infections, and drainage of the abscess through the skin can alleviate pain and swelling [6]. Detailed history taking of dental caries and periodontal disease, old teeth trauma, and endodontic treatments can help identify the dental origin. A careful oral examination that identifies severely decayed teeth or teeth with crowns also suggests that the cause of the lesion could be of dental origin [14].

Once diagnosed with a cutaneous odontogenic sinus, treatment of the causal tooth should be considered first. Endodontic treatment or surgical root canals and apicoectomies for restorable teeth and extraction of non-vital teeth can eliminate infection. The sinus tract will typically resolve within 2 weeks, while minimal dimpling and hyperpigmentation can remain [6]. Surgical revision for scar tissue can be delayed after resolution of the lesion [15]. Surgical intervention for dimpling is also possible immediately before and after treatment of causal teeth without recurrence.

\section{CONFLICT OF INTEREST}

No potential conflict of interest relevant to this article was reported.

\section{PATIENT CONSENT}

The patients provided written informed consent for the publication and the use of their images.

\section{ORCID}

Lan Sook Chang https://orcid.org/0000-0003-4725-772X

\section{REFERENCES}

1. Jones KB, Morcuende JA, DeYoung BR, El-Khoury GY, Buckwalter JA, Dietz FR. Unusual presentation of lipoblastoma as a skin dimple of the thigh: a report of three cases. J Bone Joint Surg Am 2004;86:1040-6.

2. Cohen PR, Eliezri YD. Cutaneous odontogenic sinus simulating a basal cell carcinoma: case report and literature review. Plast Reconstr Surg 1990;86:123-7.

3. Spear KL, Sheridan PJ, Perry HO. Sinus tracts to the chin and jaw of dental origin. J Am Acad Dermatol 1983;8:486-92.

4. Yasui H, Yamaguchi M, Ichimiya M, Yoshikawa Y, Hamamoto Y, Muto M. A case of cutaneous odontogenic sinus. J Dermatol 2005;32:852-5.

5. Gulec AT, Seckin D, Bulut S, Sarfakoglu E. Cutaneous sinus tract of dental origin. Int J Dermatol 2001;40:650-2.

6. Kaban LB. Draining skin lesions of dental origin: the path of spread of chronic odontogenic infection. Plast Reconstr Surg 1980;66:711-7.

7. Gupta R, Hasselgren G. Prevalence of odontogenic sinus tracts in patients referred for endodontic therapy. J Endod 2003;29: 798-800

8. Herd MK, Aldridge T, Colbert SD, Brennan PA. Odontogenic skin sinus: a commonly overlooked skin presentation. J Plast Reconstr Aesthet Surg 2012;65:1729-30.

9. Gupta M, Das D, Kapur R, Sibal N. A clinical predicament: diagnosis and differential diagnosis of cutaneous facial sinus tracts of dental origin. A series of case reports. Oral Surg Oral Med Oral Pathol Oral Radiol Endod 2011;112:e132-6.

10. Smith EL, Petty AH. Chronic dental sinus of unusual location. Br J Dermatol 1962;74:450-3.

11. Strader RJ, Seda HJ. Periapical abscess with intranasal fistula. Oral Surg Oral Med Oral Pathol 1971;32:881-4.

12. Pasternak-Junior B, Teixeira CS, Silva-Sousa YT, Sousa-Neto 
MD. Diagnosis and treatment of odontogenic cutaneous sinus tracts of endodontic origin: three case studies. Int Endod J 2009;42:271-6.

13. Obayashi N, Ariji Y, Goto M, Izumi M, Naitoh M, Kurita K, et al. Spread of odontogenic infection originating in the maxillary teeth: computerized tomographic assessment. Oral Surg Oral Med Oral Pathol Oral Radiol Endod 2004;98:223-31.
14. Cantatore JL, Klein PA, Lieblich LM. Cutaneous dental sinus tract, a common misdiagnosis: a case report and review of the literature. Cutis 2002;70:264-7.

15. Barrowman RA, Rahimi M, Evans MD, Chandu A, Parashos P. Cutaneous sinus tracts of dental origin. Med J Aust 2007;186: 264-5. 